\title{
Wheel rim state assessment using modal and geometrical parameters
}

\author{
Arkadiusz Rychlik ${ }^{1, *}$ \\ ${ }^{1}$ University of Warmia and Mazury in Olsztyn, Faculty of Technical Sciences, Department of \\ Vehicle and Machine Construction and Operation, Oczapowskiego 11, 10-719 Olsztyn, Poland
}

\begin{abstract}
This paper presents the concept of a non-invasive method for assessing the wheel rim state to determine its technical status. The research procedure includes an analysis of the wheel rim frequency spectrum in a trial at a diagnostic station, which involves applying vibration forces with specific parameters on the wheel rim. The distribution of the wheel rim's natural frequencies in rotation angle and unbalanced value with its positioning and radial run-out and axial run-out for the wheel rim bead in relation to the wheel disc centring hole on the internal and external wheel rim surfaces, are established based on the analysis and calculations. These parameters also serve to determine the condition of joints (fusion-welded, pressure-welded, etc.) and to identify the cracks or loss of integrity in the wheel rim structure. This method can be applied for tests of both new wheel rims during the quality control and used rims used for passenger cars, special vehicles and slow-running vehicles.
\end{abstract}

\section{Introduction}

Cold-formed sections are the most commonly used structural elements in engineering and technology, for instance, in simple constructions for manometric connectors on pipelines, photovoltaic or solar panel installation systems and traffic signs or banner ad holders. Examples of formed sections also include more complex and critical elements, e.g. plane wings and propeller blades or wheel rims.

Since wheel rims play a crucial role for the driver's safety and vehicle's operation, they should be periodically assessed during both the manufacturing process and operation. However, this approach is only followed in motorsports as the driver's and audience's safety is most important. In professional car and motor racing teams, qualified and experienced technicians perform visual checks of the complete wheels before they are assembled on vehicles or Formula One cars. The teams monitor the total number of driving hours for individual wheel rims and check their geometrical parameters before each assemblage. They use ultrasound and penetration methods to detect cracks and structural changes that are invisible to the naked eye. A wheel rim with any sign of fatigue or damage is scrapped and replaced with a new one. In fact, technical referees during Indy Car or F1 races require the teams to comply with strict standards for new wheel rims and to monitor the period in which the wheel rims are in operation. According to the technical regulations of such associations, used wheel rims should be scrapped every 500 miles $(800 \mathrm{~km})$. A similar rule applies to road races although the limitations to the period in operations are not that restrictive.

Unfortunately, such rigorous control procedures are uncommon among users and in the legal regulations on the vehicles involved in standard road traffic. For these type of vehicles, the condition of the wheel rim and the wheel is assessed during seasonal type replacement in tyre service points where a technician basically visually assesses the degree of whole wheel run-out and rarely the wheel rim alone while balancing the whole wheel.

* Corresponding author: arkadiusz.rychlik@uwm.edu.pl 
Importantly, assembling and disassembling of wheel rims is not beneficial since this procedure negatively mainly affects the wheel disc by provoking accelerated wear and tear of screw holes and alignment hole.

It should be noted that the wheel disc and hoop fatigue phenomenon results from a combination of cyclicity and intensity of load to which the wheel rims are exposed. While small loadings are repeated thousands of times (cycles), substantial loads accelerate the fatigue process and the number of cycles till the structure is damaged (cracked) is significantly reduced [1].

All-wheel rims are subject to major deformations when a vehicle takes sharp turns. During standard road traffic or off-road driving, the magnitude of wheel rim and hoop deformation is small. Since modern tyres have almost the same operational parameters as the former racing tyres, each turn taken at the limits of wheel grip and stability of a vehicle's driving track results in substantially greater deformations of the wheel rim fragments. Furthermore, wheel spins (applying motor power allowance in relation to the wheel grip), driving over the edges of hard objects (kerbs, ruts, faults), off-road driving and the operation of vehicles at their maximum speed and load (the phenomena of bodywork movement dynamics) should all be considered, as all of this translates into additional forces acting on the wheel rim. When a vehicle moves in this way, the wheel rims often reach temperature levels that are uncommon for standard operational conditions since they contribute to dissipating heat generated during braking (frictional parts of the braking unit) and for electric drive vehicles - also from cooling of the drive system situated inside the wheel rim [2], which is very challenging for the wheel rims of such wheels. These constant cycles of changes, from environmental temperature, dynamics of longitudinal and crosssectional loads to operational conditions and the goal of reducing the mass of wheel rims, may accelerate and limit the life durability of a wheel rim.

The methods of monitoring the structures and objects to identify and predict their conditions have become a major research area that is called NDT (Non-Destructive Testing). Damage to the structure and elements of machines can be prevented by detecting the cracks early, both those resulting from fatigue, with different non-destructive testing methods. Standard non-destructive testing techniques, such as penetration tests, magnetic methods, ultrasound, etc., have their intrinsic limitations and are often expensive and provide inconclusive feedback on the condition. During the next decade, a number of alternative methods based on identifying the nature and parameters of frequencies may become an effective, fast and handy diagnostic tool to detect the lack of structural integrity in machine parts and design systems.

It is commonly believed that the vibration theory is correlated with the modal parameter of a system, i.e. with natural frequencies, damping and types of vibrations. In other words, this is a physical system composed of the physical properties of a construction (mass, stiffness, and damping). These modal parameters are homogeneous systems described with differential equations of a model's physical motion that are expressed in relation to its mass, damping and stiffness, acceleration, velocity and displacement. Therefore, all changes in the modal parameters are conditioned by the physical features of the modelled object, which is due to damage.

Of these methods and diagnostic tools, the following should be mentioned: identification of dynamic characteristics such as natural frequencies (NF) [3], mode shapes and their derivative parameters [4], time domain vibration response [5-8] frequency response functions [9], power spectral density [10], transmissibility functions [11], coherence functions [12], correlation functions [13], etc., which serves to identify the condition of an investigated object based on emitted vibration signals.

This paper presents a comprehensive diagnostic method for identifying the condition of wheel rims not only in their modal (wheel rim structure parameters) or dynamic 
(assessment of unbalance and its positioning) aspects, but also with regards to the geometry (distribution of radial run-out and axial run-out for the wheel rim bead in relation to the wheel disc centering hole). The proposed methodology can be used to determine the optimal diagnostic signals and features that, after disassembling, help to identify the types and nature of damage to the ' 1 wheel rims that occurred during operation, whereas in the future it will be applied to diagnose the complete wheel on the vehicle with the principles of the SHM (Structural Health Monitoring) philosophy.

\section{Methods and experimental}

In general, the method used to determine the condition of wheel rims consists of identifying the nature of natural frequencies (NF) with respect to the time and frequency for which the selected features of signal are determined and compared to the general reference model typical of a given type and model of wheel rim.

The analyses of damage to the wheel disc revealed that the phenomenon of inadequate join penetration between the wheel disc and the hoop results in a lack of connection between one or several wheel disc arms with the hoop. For new wheel discs, this issue is mainly an effect of incorrect welding parameters during the manufacturing process. It is emphasised that this type of non-operational state is difficult to detect and does not disqualify the wheel rim from operation; the wheel rim is most often damaged when it is overloaded or during extreme operating conditions. A similar phenomenon is also found for the wheel rims with a specific mileage. Loss of quality in the connections between the wheel disc and the hoop may be caused by a fatigue crack or an overload crack, a change in the structure's properties due to different phenomena, such as repairs (e.g. levelling), corrosion of fusion weld or materials of the wheel disc or hoop. The geometry of the wheel rims in relation to the wheel disc opening is very important for the safety and durability of the entire wheel operation.

At the initial stage, the objective was to develop a non-invasive method of assessing the condition of disc wheel rims after their manufacturing process. Verification tests of the developed method and the diagnostic station confirmed the efficacy and usability of the proposed methodology and, thus, it was also applied for the used wheel rims, i.e. ones that were already in operation.

\subsection{Research objective}

According to the developed method, the objective of diagnostic studies was to identify the following wheel rim parameters:

- loss of integrity or structural cracks that are assessed based on changes in stiffness and/or wheel rim damping (however, the damping phenomenon is not covered in this paper) for fusion-welded, pressure-welded, glued or riveted connections made of steel, steel cast or aluminium;

- geometrical parameters, such as radial run-out and axial run-out for the wheel rim bead in relation to the wheel rim cantering hole;

- dynamic parameters: balance quality factor, i.e. identifying the heaviest and lightest side of the wheel rim and its marking.

\subsection{Subject of research}

The studies were carried out on car and working machine wheel rims of discs, in different dimensions and of varied types, both new and already in operation. The wheel discs were all joint with the hoop via the arms, i.e. with the so-called "sequential bonding". 


\subsection{Diagnostic station and equipment}

The nature of the ZRTOK diagnostic station, together with all major components, is presented in Figure 1. This station was constructed based on a serial balancing machine for vehicle wheels.

The identification of wheel rim modal parameters involves analysing the vibration signal recorded by the measuring sensors (4) situated on the shaft keeps frame (2). The measuring process begins with mounting a tested wheel rim (1) on the centring holder and fixing it with the pressure nut (3), similarly to the case of wheel mounting on a wheel balancing machine. When in such position, the wheel rim is exposed to vibration input (surge force) via a jackhammer (6) or via a mechanical vibration exciter. The vibrationexciting strokes are located on the edge of the wheel rim, at its lowest point, perpendicularly to the hoop. This process is repeated cyclically, every $90^{\circ}$ of the hoop turn, to identify the damping and stiffness parameters for the wheel disc. This measurement generates four amplitude-time runs for each position of the wheel rim $\left(0^{\circ}, 90^{\circ}, 180^{\circ}, 270^{\circ}\right)$ that are determined by the angle of rotation sensor (5). The angle of rotation for the wheel rim during the next measurement may have any value and mainly depends on the number of wheel disc arms connected with the wheel hoop.

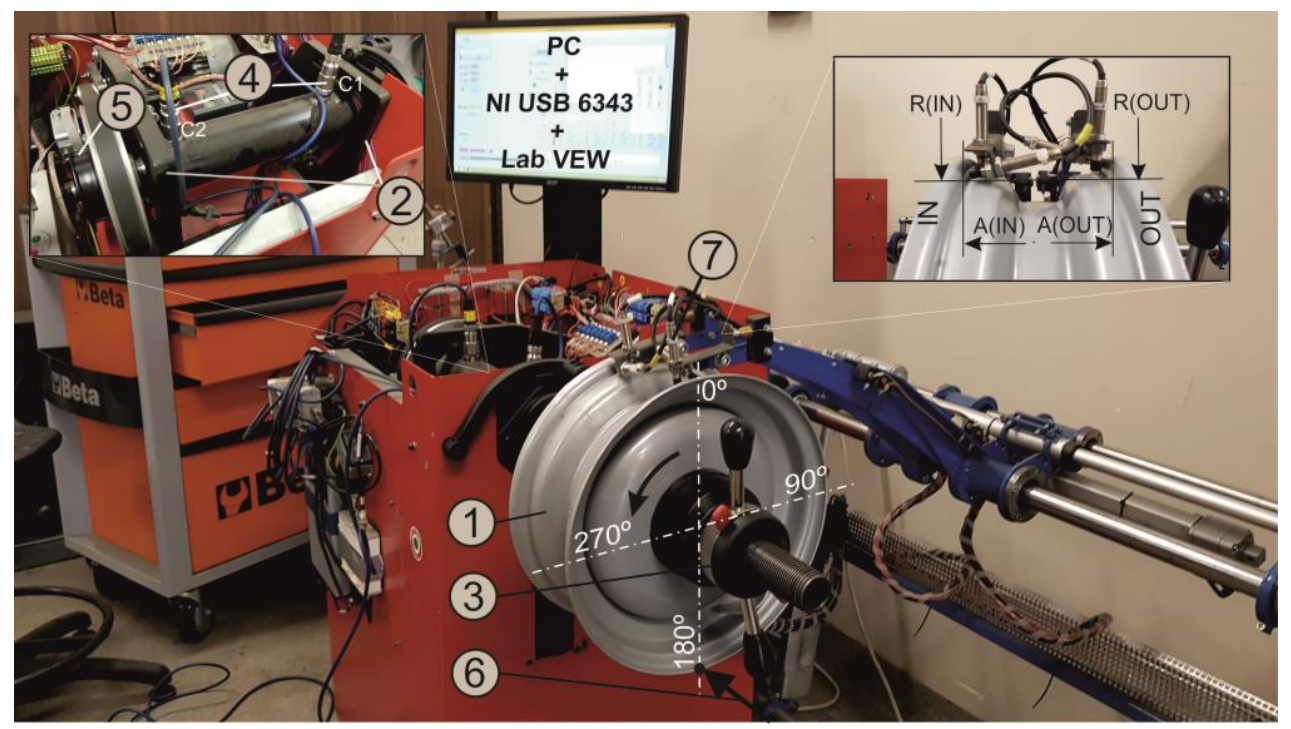

Fig.1. The components of the ZRTOK diagnostic station: 1- an investigated wheel rim; 2 - frame with the shaft to assemble a wheel rim; 3 - pressure nut for the wheel rim; 4 - vibration acceleration sensors ( $\mathrm{C} 1$ - the front one, $\mathrm{C} 2$ - the rear one); 5 - angle of rotation sensor (encoder); 6 - vibration exciter; 7 - wheel rim bead radial run-out and axial run-out measuring headers. The shaft rotation drive system and head positioning system are not presented in the drawing.

The value of rotation angle for the shaft on which the wheel rim is mounted is referred to as the hoop valve hole. When vibrations are excited and registered, the hoop is immobilized (it does not turn). Based on the analysis of time runs recorded by two sensors for four positions of the wheel rims, a damping factor is determined and following a FFT analysis, an vibration frequency spectrum is generated for each position and used to analyse the modal parameters of the hoop, among others.

Measurements of the wheel rim's geometrical parameters (as the fifth measurement) is run at the same hoop positioning as for the modal parameter testing, yet before the measurement is initiated, the automated system will move the measuring sensors (7) to the wheel rim bead and the drive unit then performs several full wheel rim turns. Meanwhile, the sensors record the length parameters recorded for the axial (A) and radial (R) plane and 
for the internal (IN) and external (OUT) wheel rim bead in relation to the wheel rotation angle. This approach serves to determine the maximum and minimum axial and radial runout for the internal and external wheel rim bead. The results of these measurements and calculations may be reflected on the wheel rim by putting a spot or other appropriate marking.

In a similar way, using the phase angle method, the "light" and "heavy" side of the wheel rim is determined. The only difference is the application of acceleration sensors and a shaft rotation angle sensor. The total time of all the measurements regardless of the type or wheel rim dimension is approx. $20 \mathrm{~s}$, not including the mounting and removal of wheel rim on or off the shaft of the diagnostic station.

The ZRTOK measuring system is equipped with two piezoelectric acceleration sensors (ICP 100 series $-100 \mathrm{mV} / \mathrm{g}$ ) and a shaft position sensor - encoder with 8-bit resolution $\left(1,40^{\circ}\right)$. Four OP-200-CW-10/80 eddy-current tracks are used as the axial run-out and radial run-out sensor, while the Impulse Force Hammer model: 086D05 (PCB) serves as a vibration exciter.

The measuring process of signal and working apparatus movements (i.e. measurement induction, wheel rim turns, positioning of wheel rim geometry measuring head) is automated and controlled by the NI USB 6343 card from the LabVIEW level.

\section{Results and discussion}

\subsection{Course of test trials}

The test trials of the wheel rim were conducted according to the following schedule: when the wheel rim was installed on the station shaft in relation to the valve hole position, a hammer stroke on the wheel rim was manually induced (the stroke force: $100 \mathrm{~N} \pm 10 \mathrm{~N}$ ). Preliminary experiments confirmed the repeatability of diagnostic signals regardless of the sample of rim fixing, providing there was no damage to a wheel disc cantering hole and the procedure of mounting was complied with the requirements of the balancing machine manufacturer. Together with a hammer motion (the stroke), a procedure of recording the diagnostic signals was initiated, which lasts approximately 2 seconds at $2 \mathrm{kHz}$ sampling frequency per channel for each angular position of the wheel rim.

\subsection{Trial results and analysis}

The time runs and acceleration frequency runs were generated during the trials and the values were quantitatively analysed. The selected results of these analyses are depicted below.

Based on the time runs for each of the measurements, the damping factor was determined and its value and change in relation to the angle of rotation for the wheel rim is a measure of wheel rim structure damping in the identified directions. The logarithmic decrement is defined as the ratio of absolute values for two consecutive extreme deflections. A change in this parameter may result from such issues as a lack of symmetry of the hoop due to its damage (e.g. deformation) or a lack of integrity of wheel rim material structure or the connection between the wheel disc and the hoop. The issue of damping decrement in the identification of the technical condition of wheel rims will not be further presented here due to the restrictions as to the length of this article.

The next stage of the analysis involves analysing the time runs with the Fourier transform. Figure 2 presents the frequency runs for the individual wheel rim rotation angles of a new 5Jx13H2 (material light alloy) wheel rim, together with the selected frequencies characteristic of the diagnostic station and the tested rim, recorded by the $\mathrm{C} 1$ sensor (Fig.1).

The analysis of the runs presented on the vibration spectrum allows distinguishing two organized groups of peaks. The first one is associated with the design of the ZRTOK diagnostic station and the other with the tested wheel rim. The spectrum visualized in 
Figure 2 features the resonant frequency for the station shaft on which the tested wheel rim was mounted (labelled: NF of Shaft ZRTOK) and vibration frequency of the frame on which the shaft of positioned (NF of Frame ZRTOK) and their next harmonics. The following frequencies are associated with the tested wheel rim: natural frequencies of the complete wheel rim (NF of WHEEL RIM), natural frequencies of the hoop (HF of HOOP (Sound of Hoop)) and its next harmonics at high frequencies (not visible in the plot).

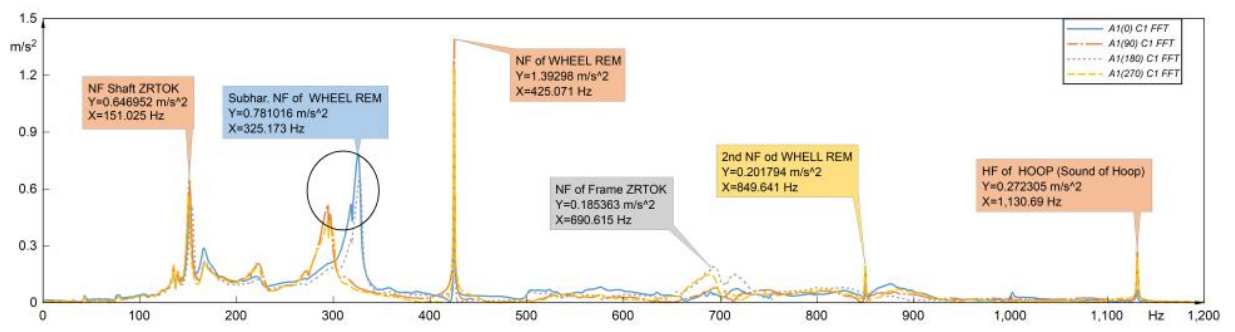

Fig. 2. The vibration amplitude spectra for the new $5 \mathrm{Jx} 13 \mathrm{H} 2$ wheel rim, recorded on the ZRTOK diagnostic station; the selected frequencies characteristic of the station and the tested wheel rim are emphasized for the following four measurement angles: $0^{\circ}, 90^{\circ}, 180^{\circ}, 270^{\circ}$.

When comparing the obtained frequency spectrums for the particular measurement angles of the tested wheel rim (Fig. 2), it could be concluded that the spectrums are similar (they overlap each-other) in the characteristic points (PEAK). This is confirmed by a comparative analysis of both runs, based of which it was found that the max relative error in the analysed characteristic points does not exceed $0.5 \%$ for the frequency values, and it does not exceed $15 \%$ for the vibration amplitude. Such a large relative error of the vibration amplitude resulted from a manual method of vibration excitation.

In order to differentiate the vibration form of the station and the tested wheel rim, a time frequency spectrum of the $5 \mathrm{Jx} 13 \mathrm{H} 2$ wheel rim (the same, whose vibration spectrum was presented in Fig. 2) was used (Fig. 3). The frequencies related to the diagnostic station are characterised by a high damping of vibration amplitudes. On the other hand, the vibration amplitudes at the frequencies related to the tested wheel rim, are much longer due to significantly lower damping of material and structure of the wheel rim compared to the damping of the station's construction. During the preliminary studies of the method and the diagnostic station, a detailed identification of these frequencies (mod), with both experimental method using the vibration signal and the acoustic signal and the obtained study results were confirmed with the analytic and simulation method.

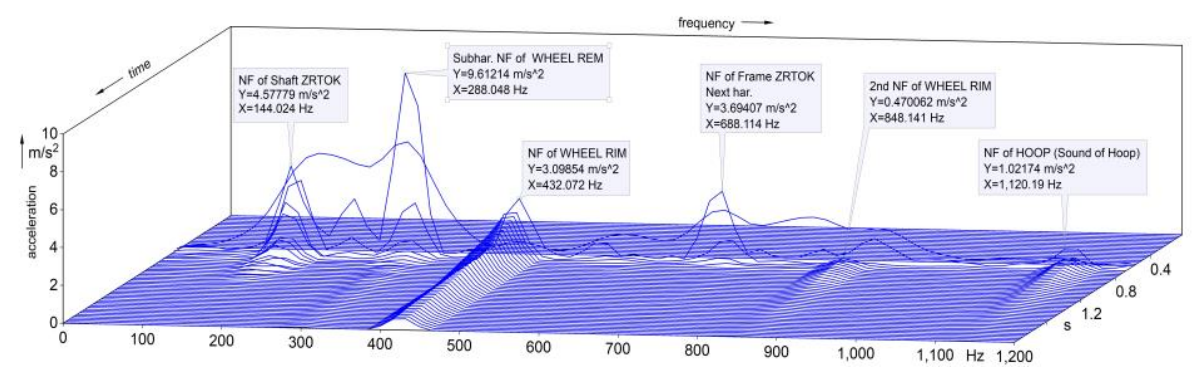

Fig. 3. Time frequency spectrum recorded by sensor $\mathrm{C} 1$ for the $5 \mathrm{Jx} 13 \mathrm{H} 2$ wheel rim, position angle of the tested wheel: $0^{\circ}$, description in the text.

For the discussed method, the nature of evaluation and identification of the condition of a tested wheel rim is based on comparing the recorded time and frequency runs to the angle of rotation for a tested wheel rim during measurements. If the wheel rim has similar 
geometric and structural properties and a quality connection between the wheel disc and the hoop at each of the investigated directions, the runs are similar to a wheel disc

Based on an analysis of the study results and identification of diagnostic signals the following sets of wheel rim unfitness symptoms were distinguished at the ZRTOK diagnostic station:

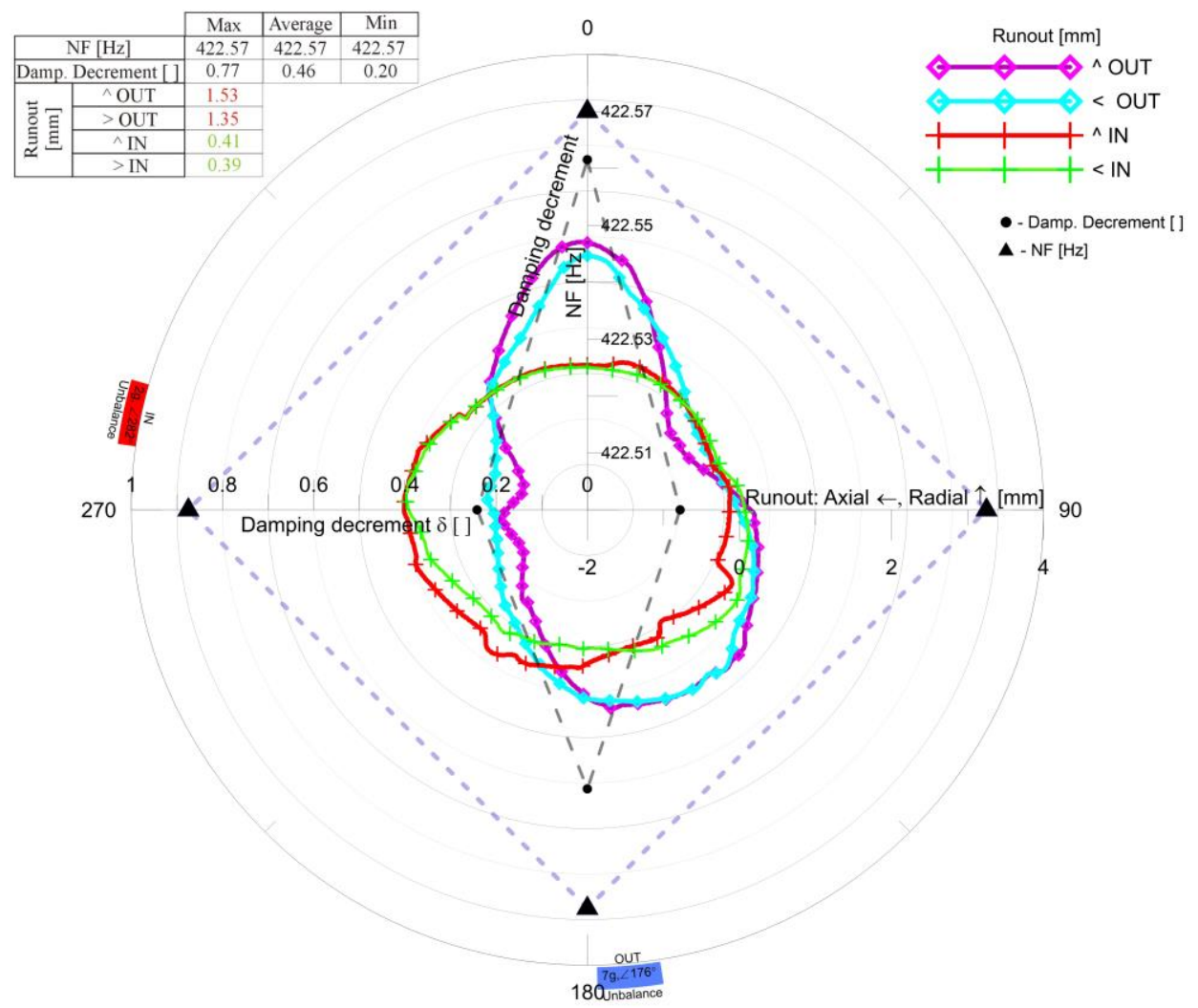

Fig. 4. The graphical report of the 5Jx13H2 wheel rim test trials (manufacturing date: 1993), generated by the ZRTOK system.

Spectral asymmetry - understood as the value of standard deviation or relative error of $\mathrm{NF}$ and the vibration spectrum in the characteristic points in relation to subsequent measurements $\left(0^{\circ}, 90^{\circ}, 180^{\circ}\right.$ and $\left.270^{\circ}\right)$ for the same wheel rim. Examples of damage escribed by this feature: rim material crack, faulty connection of a rim with a disc, etc.

Damping decrement asymmetry - defined as a "high" value of the damping decrement average $(\delta)$ and a "low" value of damping decrement standard deviation $\left(\mathrm{SD}_{\delta}\right)$ for subsequent measurements of a tested wheel rim. Examples of damage: wheel rim structure discontinuity, varied directional stiffness of a wheel rim.

Faulty mounting on the ZRTOK station shaft or a damage to a centring hole of the wheel disc - described as a "low" value of an average damping decrement $\delta$ and a "low" value of $\mathrm{SD}_{\delta}$, for subsequent measurements of a tested wheel rim as well as a "low" value of the amplitude of the recorded vibrations.

High frequency spectral asymmetry (within the frequencies of rim sounds - sound of Hoop) - understood as a formation of a series of neighbouring peaks (organised amplitudes) at the wheel hoop sound frequencies. This phenomenon is caused by 
discontinuity of a butt weld structure of the wheel hoop (e.g. cracking during spin-forming or operation resulting from a lack of re-melting during wheel welding).

Geometric asymmetry - exceeding the permissible value of radial and axial run-out of the internal (IN) and external (OUT) side of the wheel rim bead relative to a centring hole of a wheel disc with marking in respect of the norms requirements.

Good balancing - identifying the correction mass value in g (grams) reduced to the diameter of wheel rim necessary to dynamically balance a wheel rim for each of the sides including marking in respect of the norms requirements.

Permissible values and parameter limit values for conditions: NF, damping decrement, run-out value or good balancing are individualised and connected with a mass, dimension, kind and type of a tested wheel rim and the requirements imposed by a producer or a technical norm.

A two-state classification of conditions (fit, unfit), identical for all the symptoms with an unconditional differentiation of conditions was used in the developed diagnostic model of wheel rim technical condition identification. Figure 4 presents a graphical report (spider diagrams) of a $5 \mathrm{Jx} 13 \mathrm{H} 2$ wheel rim test trials (the wheel rime is made of light alloy, manufacturing date: 1993) that was generated by the ZRTOK system at the end of the test trial procedure.

The analysis of the results also reveals a constant value of natural frequencies (NF) for each measured angle of the wheel rim. For the geometry and damping factor, asymmetry becomes obvious. According to the standards, the value of axial run-out and radial run-out of the tested wheel rim on the OUT side eliminates the wheel rim from further operation. This geometry asymmetry of the external (OUT) side of the wheel rim includes the distribution of the damping factor resulting from other damping of the wheel rim at $0-180^{\circ}$ and $90-270^{\circ}$ directions.

\section{Conclusions}

Based on the test trials and the analysis of discussed method and diagnostic station, the following conclusions are presented:

- The discussed methodology and the diagnostic station is applied both for identifying the unfitness of wheel rims, such as a lack of design integrity or structural integrity of the wheel rim caused by a crack, corrosion or mechanical wear and other issues making the wheel rims unfit for use, for instance, unbalance and geometrical asymmetry of wheel rim;

- The assessment of amplitude-frequency runs for the subsequent angle measurements of the wheel rim allows identifying the condition of connection between the wheel disc and the hoop. Significant spread of NF values for the subsequent measurements is the main feature of the signal indicating this type of damage. However, it should be considered that a similar symptom is caused by substantial asymmetry of the wheel rim or a lack of structural integrity of the wheel rim.

- The condition of centring the hole of a wheel rim has a substantial impact on measurements of modal parameters; all defects, damages (cracks) and additional centring sleeves (often included in wheel rims made of light alloys) significantly limit the accuracy of measurements and the quality of results analyses, therefore for the purpose of the presented diagnostic method, an identification procedure for such a condition was developed in the form of a faulty mounting or damage to the centring hole of the tested rim;

- The increment of measuring angle (number of measurements) for the individual diagnostic signals can be reduced to $20^{\circ}$ to perform more precise analyses of the wheel rim condition. A smaller value of increments for the wheel rim rotation angle than the proposed one does not impact the quality or informative nature of the recorded values; 
- The discussed research methodology can be applied for identifying the modal parameters of new wheel rims (verification of the manufacturing process quality - the method is used in the quality control system for the end-products in the disc wheel rims manufacturer Polkar Warmia) and wheel rims that are already in operation in order to identify their condition, e.g. during a seasonal tyre exchange in a tyre exchange station.

\section{Acknowledgments}

This research was conducted under NCBR project no. POIR.01.01.01-00-1746/15 by POLKAR Warmia Sp. z o., ul. Fabryczna 21, 11-040 Dobre Miasto.

\section{References}

1. J. K. Sinha, M. I. Friswell, S. Edwards, Simplified models for the location of cracks in beam structures using measured vibration data. Journal of Sound and Vibration 251(1), p. 13-38. (2002). DOI: 10.1006/jsvi.2001.3978

2. Matej Biček, Gorazd Gotovac, Damijan Miljavec, Samo Zupan. Mechanical failure mode causes of in-wheel motors. Strojniški vestnik - Journal of Mechanical Engineering, Vol. 61, p. 74-85. (2015). DOI:10.5545/sv-jme.2014.2022

3. K. He and W.D. Zhu, Structural damage detection using changes in natural frequencies: theory and applications. Journal of Physics: Conference Series, Vol. 305, Issue 1, p. 012054. (2011)

4. N. M. M. Maia, J. M. M. Silva, E. A. M. Almas, Damage detection in structures; from mode shape of frequency response function methods. Mechanical Systems and Signal Processing, Vol. 17, Issue 3, p. 489-498. (2003)

5. J. Cattarius, D. J. Inman, Time domain analysis for damage detection in smart structures, Mechanical Systems and Signal Processing, Vol. 11, Issue 3, p. 409-423. (1997)

6. T. Kuroiwa, H. Iemura, Vibration based damage detection using time series analysis, The 14th world conference on earthquake engineering, Beijing, China, October 12-17. (2008)

7. A. Rychlik. Detection of structural damage in vibroacoustic analysis. Journal of KONES Powertrain and Transport, Vol. 23, No. 1, p. 279-288. (2016).

8. A. Rychlik, A method for evaluating the technical condition of wheel rims in slow moving vehicles based on modal parameters. DIAGNOSTYKA, Vol. 17, No. 2, p: 65$70,(2016)$

9. N. Guo, Z. Yan, Y. Jia , L. Wang. Model updating using correlation analysis of strain frequency response function, Mechanical Systems and Signal Processing, Vols. 70-71, p. 284-299. (2016)

10. Z. D. Zheng, Z. R. Lu, W. H. Chen, J. K. Liu., (2014). Structural damage identification based on power spectral density sensitivity analysis of dynamic responses, Computers \& Structures, Vol. 146, p. 176-184. DOI: 10.1016/j.compstruc.2014.10.011

11. D. Zhu, Yi X., Y. Wang. Sensitivity analysis of transmissibility functions for structural damage detection, Proceedings of SPIE 7983, (2011) 79832M-1, DOI: $10.1117 / 12.879867$

12. D. D. Rizos, S. D. Fassois, Z. P. Marioli-Riga, A. N. Karanika, Vibration-based skin damage statistical detection and restoration assessment in a stiffened aircraft panel. Mechanical Systems and Signal Processing, Vol. 22, Issue 2, p. 315-337. (2008) 
13. M. Zhang, R. Schmidt, Sensitivity analysis of an auto-correlation-function-based damage index and its application in structural damage detection. Journal of Sound and Vibration, Vol. 333, Issue 26, p. 7352-7363. (2014) 